\title{
Resección quirúrgica de malformación arteriovenosa de alto flujo en ángulo nasogeniano con embolización preoperatoria. Reporte de un caso
}

\section{Surgical resection of high-flow arteriovenous malformation of the nasogenian sulcus with preoperatory embolization. A case report}

\author{
Lara Fernández $\mathbf{R}^{\mathbf{1}}$, Paul Boettiger $\mathbf{B}^{2}$, Héctor Bahamonde $\mathbf{S}^{\mathbf{2}}$.
}

\begin{abstract}
RESUMEN
El 50\% de las malformaciones arteriovenosas (MAVs) de alto flujo se ubican en la región craneofacial y pueden producir sangrado con riesgo vital sin diagnóstico y tratamiento adecuado. La resonancia magnética (RM) es útil en el diagnóstico diferencial de las lesiones vasculares, siendo el gold standard la angiografía selectiva. Dado que las MAVs de alto flujo no involucionan espontáneamente, el tratamiento usualmente consiste en embolización con posterior resección quirúrgica.

Se presenta un paciente de sexo masculino de 20 años que consulta por aumento de volumen nasogeniano derecho, progresivo, violáceo, blando y pulsátil. Se objetiva mediante RM una zona de vasos serpentiginosos, que se extiende desde el subcutáneo hasta la pared anterior del maxilar. La angiografía cerebral confirma MAV de alto flujo e informa mapeo vascular. Se decide embolización prequirúrgica superselectiva con posterior resección tumoral mediante acceso transvestibular.

Palabras claves: Malformación arteriovenosa (MAV), alto flujo, embolización superselectiva.
\end{abstract}

\begin{abstract}
The $50 \%$ of arteriovenous malformations (AVMs) of high flow are located in the craniofacial region and can cause life-threatening bleeding without a diagnosis and appropriate treatment. Magnetic resonance imaging is useful in the differential diagnosis of vascular lesions, being the gold standard selective angiography. Since high-flow AVMs do not regress spontaneously, treatment usually involves surgical resection with subsequent embolization.

We present a 20 years male patient consulting with a purple, progressive, soft, pulsating enlargement of right-side nasogenian region. Magnetic resonance imaging shows serpiginous vessels, extending from the subcutaneous to the anterior wall of the maxillary. Cerebral angiography confirms high flow AVM and shows vascular mapping. It was decided superselective preoperative embolization with subsequent tumor by transvestibular access.

Key words: Arteriovenous malformation (AVM), high-flow, superselective embolization.
\end{abstract}

1 Interna de Medicina. Universidad de Chile.

2 Médico Servicio de Otorrinolaringología Hospital Clínico Universidad de Chile. 


\section{INTRODUCCIÓN}

En 1982, Mulliken y Glowacki clasificaron las lesiones vasculares congénitas según características histológicas y comportamiento en 2 grupos: Hemangiomas y malformaciones vasculares ${ }^{1}$. Los hemangiomas son tumores de células endoteliales que se hacen aparentes en las primeras semanas de vida, con rápida proliferación seguida de involución lenta. Las malformaciones vasculares, debidas a fallas durante la angiogénesis embrionaria², están presentes desde el nacimiento, muestran crecimiento proporcionado al volumen corporal y no muestran signos de involución espontánea ${ }^{3}$.

Las malformaciones vasculares a su vez se pueden clasificar, según sus características en la angiografía, en lesiones de bajo flujo (venosas, capilares y linfáticas) 0 de alto flujo (malformaciones arteriovenosas (MAV), arteriales y fístulas arterio-venosas) $)^{1,4}$. La importancia de las MAVs de alto flujo radica en que el $50 \%$ está localizado en la región craneofacial y pueden producir sangrado con riesgo vital sin un diagnóstico y tratamiento adecuad $0^{5,6}$.

El diagnóstico de estas lesiones se basa en características clínicas e imagenológicas. Shobinger dividió las MAV en 4 estadíos según la clínica:

- Estadio I. Enrojecimiento o aumento de la temperatura cutánea

- Estadio II. Soplo, pulsaciones audibles, lesión en expansión

- Estadio III. Dolor, ulceración, sangrado, infección

- Estadio IV. Falla cardiaca².

Otros síntomas son asimetría facial, pérdida de piezas dentales, tinnitus, cefalea y pueden verse complicaciones con riesgo vital como hemorragias, necrosis isquémica y falla cardiaca ${ }^{6,7}$.

En cuanto al diagnóstico imagenológico la angiografía selectiva se considera actualmente el gold standard pero por su costo y complicaciones, se ha limitado su uso a la determinación de la extensión, mapeo circulatorio de malformaciones, aneurismas $y$ en pacientes que requieran embolizaciones preoperatorias. La resonancia magnética y la tomografía computarizada cobran importancia dado su menor costo y complicaciones, siendo útiles en el diagnóstico diferencial de las diversas malformaciones por el patrón de contraste $^{4}$.
Dado que las MAV de alto flujo no involucionan en forma espontánea ${ }^{3}$, el tratamiento usualmente es embolización con posterior extracción de la malformación ${ }^{3,8}$. La extracción completa de la lesión es recomendable para prevenir recurrencias. La embolización tiene como objetivo ocluir el lecho de la malformación para reducir el riesgo de sangrado al momento de la resección 0 en algunos casos ser manejo único en lesiones vasculares inoperables. Sin embargo, existen potenciales riesgos de embolización de otras estructuras, vasoespasmo, ruptura de arterias aferentes dilatadas y complicaciones secundarias a cambios hemodinámicos como accidentes cerebrovasculares, isquemia de nervios craneanos, amaurosis y muerte ${ }^{3,6}$. La embolización arterial a través de una acceso transfemoral es de elección en MAV's de alto flujo $0^{9,10}$.

\section{CASO CLÍNICO}

Paciente de 20 años, sexo masculino, con antecedentes de hiperplasia adenoamigdalina de resolución quirúrgica en la infancia, consulta en Servicio de Otorrinolaringología del Hospital Clínico de la Universidad de Chile por historia de aumento de volumen con cambio de coloración de la piel en mejilla derecha, lento pero progresivo, de consistencia blanda y pulsátil, de 2 años de evolución.

Al examen físico se observa una masa en la región nasogeniana derecha, de aspecto violáceo tenue, consistencia blanda, por lo que se solicita una ecografía doppler que informa naturaleza vascular del tumor. Se complementa estudio con resonancia magnética máxilo-facial con y sin gadolinio, donde se objetiva engrosamiento de partes blandas en surco nasogeniano derecho que compromete parcialmente las partes blandas nasales y la región nasogeniana adyacente, extendiéndose desde la región subcutánea hasta la pared anterior del hueso maxilar superior derecho, presentando una zona de vasos dilatados $y$ serpentiginosos que mide $3,7 \times 2,4 \times 1,5 \mathrm{~cm}$, que realzan en fase arterial y venosa del estudio con gadolinio. En dicho informe, se sugieren como aferentes las arterias facial e infraorbitaria derecha y eferentes que drenan hacia la vena facial derecha. 
Para mapeo vascular se realiza angiografía cerebral que confirma MAV de alto flujo, con nido plexiforme aferentado por arteria facial y ramas de la arteria maxilar interna derecha, con drenaje venoso precoz principalmente a vena facial derecha y en menor magnitud por reflujo a vena oftálmica superior ipsilateral. En las Figuras 1 y 2 se muestra este examen.

Las características de la malformación y su localización justifican la realización de embolización prequirúrgica superselectiva con Spongostan ${ }^{\circledR}$ a ramas aferentes de la arteria maxilar interna y facial derecha. Con ello se logra una satisfactoria oclusión proximal de las aferentes. Las angiografías de control demuestran significativa reducción de la impregnación de la malformación. No hubo complicaciones y el procedimiento fue catalogado como exitoso.

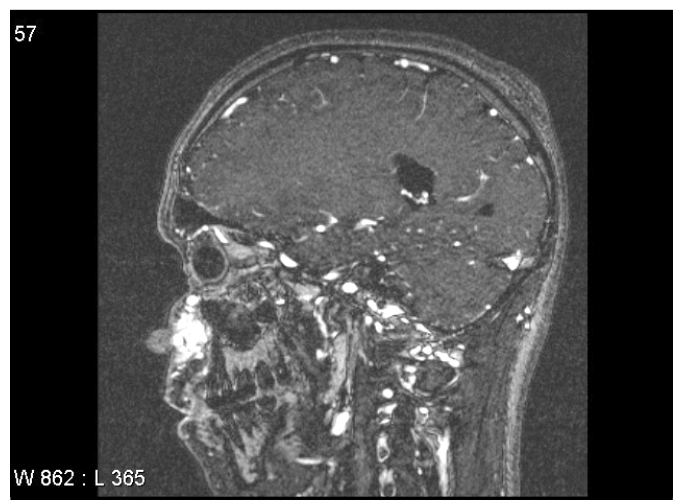

Figura 1. Reconstrucción de resonancia nuclear magnética.

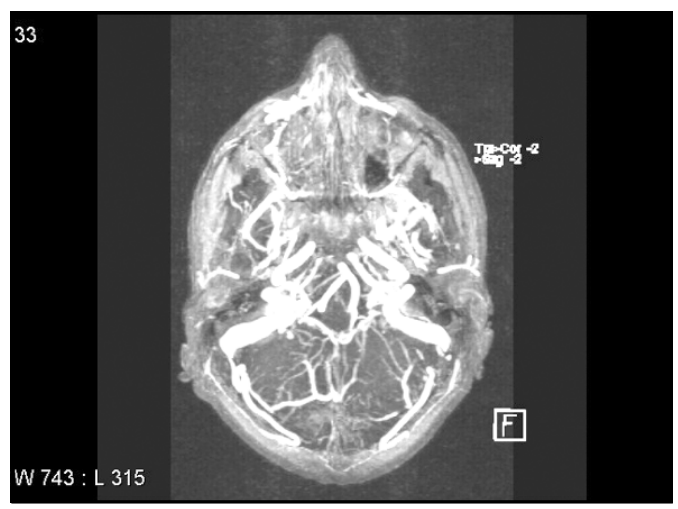

Figura 2. Arteriografía por resonancia nuclear magnética, se observa ubicación y comunicaciones del tumor.
El paciente se somete a resección tumoral a las $24 \mathrm{hr}$ de su embolización, mediante acceso transvestibular. Se realiza infiltración, se desperiostiza el seno maxilar derecho, se identifica y respeta el nervio infraorbitario y se procede a resecar delicadamente el tumor, realizando ligaduras a los múltiples aferentes arteriales y eferentes venosos, identificando como arteria principal la arteria facial, la que se liga al igual que la vena facial. Se reseca un tumor de $2,5 \times 2 \times 1 \mathrm{~cm}$, con apariencia de ovillo arteriovenoso con escaso sangrado intraoperatorio (Figura 3). Luego mediante disección con tijeras se libera la bola de Bichat en forma parcial, la que se sutura con vicryl 3.0 al lecho tumoral.

El paciente se mantiene hospitalizado por 1 día con analgesia y antibioticoterapia profiláctica, dándose de alta. Se controla a la semana y a las 2 semanas. Mantuvo evolución satisfactoria, sin signos de infección, destacando un gran edema en la zona operatoria que se resolvió a las 2 semanas y una leve paresia de la rama buccinadora del nervio facial, la que también se resolvió espontáneamente.

La biopsia informa la presencia de tejido conectivo fibroso con vasos de tipo arterial de calibre mediano con material intraluminal compatible con aneurisma arterial embolizado. El paciente se controla a los 2 meses, sin secuelas.

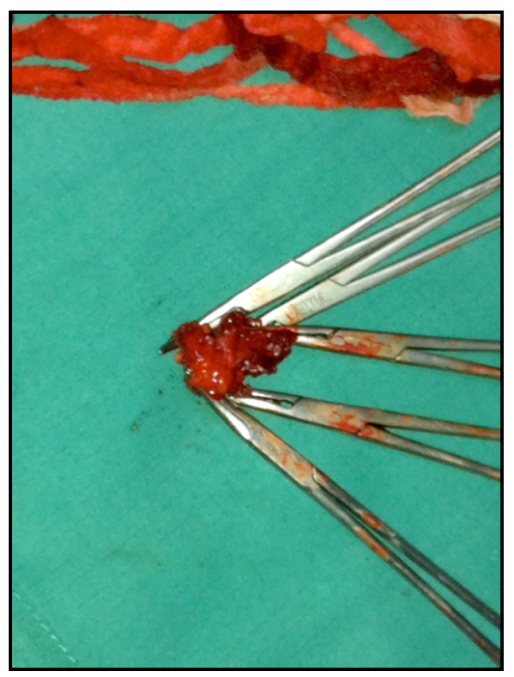

Figura 3. Tumor resecado, que muestra sus características de ovillo vascular arterio-venoso. 


\section{DISCUSIÓN}

Las malformaciones arteriovenosas craneofaciales de alto flujo son poco frecuentes y sin diagnóstico y manejo adecuado pueden producir sangrado con riesgo vital ${ }^{5,6}$. En este caso la localización y comunicantes vasculares dificultan el manejo, requiriéndose resultados estéticos y funcionales óptimos ${ }^{10}$. Se decide el manejo con embolización prequirúrgica superselectiva seguido de resección, considerado actualmente de elección por diversos autores ${ }^{5,6,8,11,12}$ y se exponen a continuación alternativas de manejo.

La evolución natural de estas lesiones puede determinar ulceración, sangrado, infección y falla cardiaca, entre otros ${ }^{2}$, por lo que al diagnóstico se debe plantear un tratamiento cuyos riesgos sean menores a los expuestos con manejo conservador y a su vez proporcione beneficios al paciente en cuestión. En este caso, la progresión de la lesión determinaría resultados estéticos poco favorables y el compromiso de estructuras faciales 0 aparición de nuevas comunicantes vasculares a territorios cerebrales más complejos podrían, en un futuro, hacer irresecable la lesión.

La resección quirúrgica como método único de manejo en la región craneofacial puede ser seguro y completo pero está asociado a hemorragias extensas y a resecciones incompletas que llevan a una reaparición de la lesión, siendo en ocasiones de mayor tamaño que la original ${ }^{5}$, causando desfiguraciones y compromiso funcional.

Por otro lado la embolización de MAVs de alto flujo como método único de tratamiento es raramente exitosa dada la aparición posterior de nuevas comunicantes vasculares a otros territorios ${ }^{5,13,14}$, además de mencionar las complicaciones propias del procedimiento con consecuencias neurológicas devastadoras para el paciente.

Para un manejo más completo y con menores complicaciones, la embolización prequirúrgica con 24 a 48 horas de antelación, como fue realizada en nuestro paciente, ha demostrado reducir la hipervascularidad con consiguiente disminución del sangrado y reduce la necesidad de reintervenciones por resección incompleta ${ }^{5}$. Este manejo multidisciplinario considerado de elección, ha mejorado el pronóstico de los pacientes con MAV de cabeza y cuello.

\section{CONCLUSIÓN}

La combinación de un diagnóstico acucioso mediante métodos imagenológicos adecuados seguido de terapia de embolización superselectiva y resección quirúrgica posterior, permanecen como alternativa segura de manejo en malformaciones ateriovenosas craneofaciales de alto flujo.

\section{BIBLIOGRAFÍA}

1. Mulliken JB, Glowacki J. Hemangiomas and vascular malformations in infants and children: A classification based on endotelial characteristics. Plast Reconstr Surg 1982; 69(3): 412-22.

2. Kohout MP, Hansen M, Pribaz JJ, Mulliken JB. Arteriovenous malformations of the head and neck: Natural history and management. Plast Reconstr Surg 1998; 102(3): 643-54.

3. Werner JA, Dünne aA, Folz BJ y col. Current concepts in the classification, diagnosis and treatment of hemangiomas and vascular malformations of the head and neck. Eur Arch Otorhinolaryngol 2001; 258(3): 141-9.

4. Ryu CW, KIm JK, KIm SJ y col. Head and Neck Vascular Lesions: Characterization of the Flow Pattern by the Use of Three-Phase CT. Korean J Radiol 2009; 10(4): 323-32.

5. Bhandari PS, Sadhotra LP, Bhargava P, Bath AS, Mukherjee MK, Maurva S. Management strategy for facial arteriovenous malformations. Indian J Plast Surg 2008; 41(2): 183-9.

6. Lemound J, Brachvogel P, Götz F, Rücker M, GeLldRICH N, EcKardt A. Treatment of Mandibular High-flow Vascular Malformations: Report of 2 cases. J Oral Maxillofac Surg 2011; 69(7): 1956-66.

7. Chen WL, Ye JT, Xu LF, Huang ZQ, Zhang DM. A multidisciplinary approach to treating maxillofacial arteriovenous malformations in children. Oral Surg Oral Med Oral Pathol Oral Radiol Endod 2009; 108(1): 41-7.

8. Jackson IT, Carreño R, Potparic Z, Hussain K. Hemangiomas, vascular malformations, and lymphovenous malformations: classification and methods of treatment. Plast Reconstr Surg 1993; 91(7): 1216-30. 
9. Giadui L, Princ G, Chiras J, Guilbert F, Bertrand $\mathrm{JC}$. Treatment of vascular malformations of the mandible: A description of 12 cases. Int J Oral Maxillofac Surg 2003; 32(2): 132-6.

10. Siniscalchi EN, Minutoli F, Catalfamo L. Intraosseous mandibular artero-venous malformations: Case report. $J$ Craniomaxillofac Surg 2003; 37(2): 106-9.

11. Coskun BU, Sozen E, Basak T, Alkan S, Dadas B. Arteriovenous malformation of the nasopharynx: a case report. Int $J$ Pediatr Otorhinolaryngol 2005; 69(9): 1287-90.

12. Pompa V, Valentini V, Pompa G, Di Carlo s, Bresadola
$\mathrm{L}$, Treatment of high-flow arteriovenous malformation (AVMs) of the head and neck with embolization and surgical resection. Ann Ital Chir 2011; 82(4): 253-9.

13. Seccia A, Salgarello M, Farallo E, Falappa PG. Combined radiological and surgical treatment of arteriovenous malformations of the head and neck. Ann Plast Surg 1999; 43(4): 35966.

14. Donnelly L, Adams D, Bisset G. Vascular malformations and hemangiomas: A practical approach in a multidisciplinary clinic. $A m \mathrm{~J}$ Roentgenol 2000; 174(3): 597-608. 